\title{
Water Tank Monitoring and Visualization System Using Smart-Phones
}

\author{
Haesung Tak, Daegeon Kwon, and Hwan-Gue Cho
}

\begin{abstract}
Monitoring sensor networks that consist of many valves, pumps and tanks is an important task for managing large ships. Existing water tank monitoring systems are only provided for PC environment. In this paper we propose a new method to monitor water tanks on a smart phone. Before presenting our system, we analyze the water tank system and define the discussed problem formally. Based on this analysis, we develop our monitoring system and elaborate on out the implementation. We also show that our system visualizes sensor data using a simple and intuitive user interface.
\end{abstract}

Index Terms-Water tank monitoring, Smartphone, monitoring system, visualization, water tank

\section{INTRODUCTION}

In recent times, smart-phones have become an important part of our daily lives. When we start to use the smart phones, we can do all-around business at anytime and anywhere. This change, known as ubiquitous computing, has been developing gradually due to smart-phones [1], [2]. In the maritime industry, communication environment is developed by sailors to know about the ship information.

Most of the typical ship-monitoring applications show the values of various sensors in the water tank. These system data classify the sensor data and store it on the server. Using this monitoring system information, we developed a new smart-phone application called "Tank boy," which will be elaborated in this paper. This system provides information from the water tank database using visualization techniques.

In this paper, first, we provide the overview of a water tank structure. In this section, we analyze sensor data typically used for water tank monitoring. In Section III, we suggest the system architecture and system flow information that will be used by the smart-phone application. In Section IV, we discuss about the issues faced by this system. Finally, Section $\mathrm{V}$, we draw conclusions and discuss future works.

\section{OVERVIEW OF WATER TANK SYSTEM}

In this paper, we monitor the real-time data processed for water tanks in ships. Before we develop the system the water tank structure needs to be analyzed [3]. Fig. 1 shows the schematic of water tank system.

Manuscript received November 25, 2012; revised January 20, 2013. This work was supported by the Ministry of Knowledge Economy(MKE), Korea Institute for Advancement of Technology(KIAT) and 00 Leading Industry Office through the Leading Industry Development for Economic Region.

The authors are with the Dept. of Computer Engineering, Pusan National University, Busan, South Korea (e-mail: \{tok33, duskan, hgcho\} (a)pusan.ac.kr).

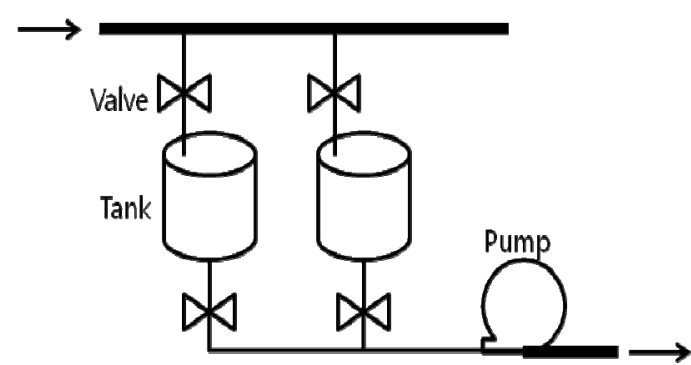

Fig. 1. Water tank system architecture

The water tank system, consist of three components: the tank, valve, and pump. The data from the sensor of each component are classified as follows.

Table I shows the data from the tank sensors. The tank consists of five sensors: high, middle and low position temperature, level, and pressure.

Table II and III show digital and analog valve sensor information, respectively. Table II depicts the input and output signals, which are control signals. Gage is indicative of the degree of valve opening and closing. Table IV shows the pump sensor data, which are input and output signals.

We develop a smart-phone application that analyzes this data, shows the state of each device', and judges whether this state is dangerous.

\begin{tabular}{ccccc}
\multicolumn{5}{c}{ TABLE I: TANK SENSOR DATA } \\
\hline \hline Name & Signal Type & Unit & Low & High \\
\hline Level & $4 \sim 20 \mathrm{~mA}$ & $\mathrm{~m}$ & 0 & 20 \\
Temp Low & $4 \sim 20 \mathrm{~mA}$ & ${ }^{\circ} \mathrm{C}$ & 0 & 200 \\
Temp Middle & $4 \sim 20 \mathrm{~mA}$ & ${ }^{\circ} \mathrm{C}$ & 0 & 200 \\
Temp High & $4 \sim 20 \mathrm{~mA}$ & ${ }^{\circ} \mathrm{C}$ & 0 & 200 \\
Pressure & $4 \sim 20 \mathrm{~mA}$ & bar & -1 & 5 \\
\hline \hline
\end{tabular}

TABLE II: VALVE SENSOR DATA (DigITAL)

\begin{tabular}{lcccc}
\hline \hline \multicolumn{1}{c}{ Name } & Signal Type & Unit & False & True \\
\hline Valve Opened & Digital Input & - & 0 & 1 \\
Valve Closed & Digital Input & - & 0 & 1 \\
Valve Open & Digital Output & - & 0 & 1 \\
Valve Close & Digital Output & - & 0 & 1 \\
Valve Fail & Digital Input & - & 0 & 1 \\
\hline \hline
\end{tabular}

TABLE III: VALVE SENSOR DATA (ANALOG)

\begin{tabular}{ccccc}
\hline \hline Name & Signal Type & Unit & False & True \\
\hline \hline Gage & $4 \sim 20 \mathrm{~mA}$ & $\%$ & 0 & 100 \\
\hline & & & & \\
& TABLE IV: PUMP SENSOR DATA & & \\
\hline Pump Remote & Signal Type & Unit & False & True \\
Pump Run & Digital Input & - & 0 & 1 \\
Pump Start & Digital Input & - & 0 & 1 \\
Pump Stop & Digital Output & - & 0 & 1 \\
Pump Fail & Digital Output & - & 0 & 1 \\
\hline \hline
\end{tabular}




\section{Proposed SMART-PhOne System}

\section{A. Proposed System Flow}

In this paper, the analysis of the water tank sensor data is used in the implementation of a monitoring and visualization system using smart-phones. The system flow, configured to implement this system, is shown in Fig. 2.

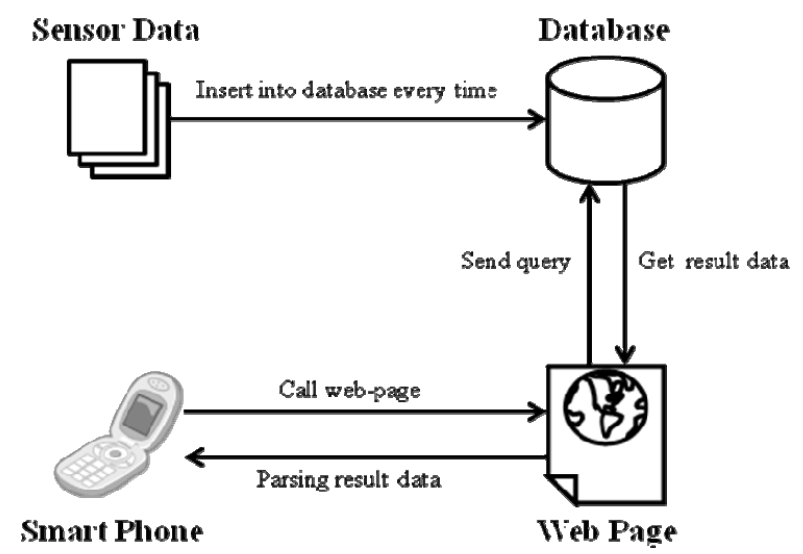

Fig. 2. System process flow

As shown in Fig. 2, the sensor data are not imported manually; the database and Web page are configured to import the data. To obtain data on particular sensors, users must go to the Web page, which request for information from the database through a database query. This query requests for information and relays this information back to the Web page. Thus, according to the system flow, the web pages obtain the query results from the database and convey them as visualization results to users.

\section{B. Database}

Smart-phones usually do not have enough memory to store real-time sensor data, which may cause substantial loads. Hence, sensor data should be stored in an external database rather than a smart-phone.

The visualization program was written in a Windows environment. This was because easy access to this environment and was provided by Microsoft for building the MySQL server, using which the database was created. The database comprised the data from the sensors as well as the analysis of these data. The database schema of the water tank, valves, and pumps are as follows.

The tank database schema is shown in Table V. By storing the time-stamp of each occurrence, unique values comprising the device name and the occurrence times can be stored on the database.

The valve database schema is shown in Table VI. This schema was constructed as one of the database tables that integrated analog and digital data shown in Tables II and III. The advantage of using a database with a single table is that it reduces the time consumed in processing data.

The pump database schema is shown in Table VII. This database connects multiple devices at the same time by adding the data for each device onto a single table.

Valves and pumps in the database to provide monitoring data that does not need to exist. This value corresponds to the digital output. In addition, these data are stored, because they may be used to predict the lifetime of the system.

In the case of large vessels, multiple devices are connected. Sensors connected to each device have different normal ranges. The database should store tag names for each device. It is used for obtaining the normal range of the sensors.

TABLE V: TANK DATABASE SCHEMA

\begin{tabular}{ccc} 
& TABLE V: TANK DATABASE SCHEMA \\
\hline \hline Name & Data Type & Purpose \\
Timk_name & Varchar & Tank tag name \\
Temp_L & Datetime & Time stamp \\
Temp_M & Double & Temperature at the bottom \\
Temp_H & Double & Temperature in the middle \\
Level & Double & Temperature at the top \\
Pressure & Double & Current Capacity \\
& Double & Tank pressure \\
\hline \hline & TABLE VI: VALVE DATABASE SCHEMA \\
\hline \hline Name & Data Type & Purpose \\
Valve_ID & Varchar & Pump tag name \\
Time & Datetime & Time stamp \\
Gage & Double & Valve opening degree \\
Opened & Integer & Open execution result \\
Closed & Integer & Close execution result \\
Open & Integer & Open Instruction \\
Close & Integer & Close Instruction \\
Alarm & Integer & Error detection \\
\hline \hline
\end{tabular}

TABLE VII: PUMP DATABASE SCHEMA

\begin{tabular}{ccc}
\hline \hline Name & Data Type & Purpose \\
\hline Pump_ID & Varchar & Pump tag name \\
Time & Datetime & Time stamp \\
Remote & Integer & Stop execution result \\
Run & Integer & Start execution result \\
Start & Integer & Start Instruction \\
Stop & Integer & Stop Instruction \\
Alarm & Integer & Error detection \\
\hline \hline
\end{tabular}

TABLE VIII: TAG LIST DATABASE SCHEMA

\begin{tabular}{|c|c|c|}
\hline Name & Data Type & Purpose \\
\hline Tag & Varchar & Tag name \\
\hline Tag_Description & Varchar & Sensor description \\
\hline Signal Type & Varchar & Physical Sensor Type \\
\hline Eng. L & Varchar & Measuring the maximum \\
\hline Eng. $\mathrm{H}$ & Varchar & Measuring the minimum \\
\hline E. Unit & Varchar & Unit of measurement \\
\hline \multicolumn{3}{|c|}{ TABLE IX: TANK TAG DATABASE SCHEMA } \\
\hline Name & Data Type & Purpose \\
\hline Tank_name & Varchar & Tank tag name \\
\hline Temp_L & Varchar & Tag name of Temp_L \\
\hline Temp_M & Varchar & Tag name of Temp_M \\
\hline Temp_H & Varchar & Tag name of Temp_H \\
\hline Level & Varchar & Tag name of level sensor \\
\hline Pressure & Varchar & Tag name of pressure sensor \\
\hline \multicolumn{3}{|c|}{ TABLE X: VALVE TAG DATABASE SCHEMA } \\
\hline Name & Data Type & Purpose \\
\hline Valve_ID & Varchar & Valve tag name \\
\hline Gage & Varchar & Tag name of gage sensor \\
\hline Opened & Varchar & Tag name of opened (Input) \\
\hline Closed & Varchar & Tag name of closed (Input) \\
\hline Open & Varchar & Tag name of open (Output) \\
\hline Close & Varchar & Tag name of close (Output) \\
\hline Alarm & Varchar & Tag name of alarm (Input) \\
\hline
\end{tabular}


TABLE XI: The PUMP TAG Database Schema

\begin{tabular}{ccc}
\hline \hline Name & Data Type & Purpose \\
\hline Pump_ID & Varchar & Pump tag name \\
Remote & Varchar & Tag name of Remote (Input) \\
Run & Varchar & Tag name of run (Input) \\
Start & Varchar & Tag name of start (Output) \\
Stop & Varchar & Tag name of stop (Output) \\
Alarm & Varchar & Tag name of alarm (Input) \\
\hline \hline
\end{tabular}

Table VIII describes the schema with the tag names of the sensors, normal measurement ranges, actual value of the incoming data, measurement units, and sensor information. Here, the sensor maintains a consolidated list of the outputs rather than that for individual sensors. Due to this, the number of sensors used in the actual ship was not large, and the decision to build only a single table was appropriate.

Table IX describes tag name of the tank sensors stored in the database table schema. The schema stores a unique tag name for the five sensors. Each sensor corresponds to one tank in the table.

Table X describes the tag name of the valve sensors stored in the database schema. Table XI describes the tag name of the pump sensors stored in the database schema. It is not different from existing table schema which is stored sensor data. But, it is useful to know what data means in the Table VIII. These databases, which are designed to consider each association, based on object oriented programming [4].

\section{Database Information Extracting}

The water-tank query result (WQR) is used for extracting information from the developed database. It is one of the modules in this system; it is actually one of the Web pages. When users request for a desired value for the devices using the query statement, the WQR processes the results. The WQR, which is seamlessly interoperable, has been developed using PHP, because sensor data is stored in a database created MySQL.

The PHP is implemented server-based program language. And then, PHP is not shown its source code for users. The user, who wants to know the information of water tank device, just input the Web page address. It is convenient because it does not complicate the receiving query results.

WQR were delivered to the information of the Web page address. The address of the Web page has the table name and the date the information needed to complete the query. When query statement complete, WQR extracts the information of water tank device from database.

\section{Smart-phone Application}

The database and WQR that can be used in an actual communication environment for the smart-phone application was designed.

According to [5], 51.8\% of smart phone users use Android, whereas $34.3 \%$ iOS.

The application development cannot be in the same language for these two operating systems. We decided to develop the application for Android due to its preference among users.

Eclipse development tools were used to create the Android applications using Java and Android API. The basic interface environment is user-friendly and simple to configure.

\section{IMPLEMENTATION ISSUE}

\section{A. Database Communication}

For Android application, SQLite is usually used to build the database. However, SQLite is not suitable for our monitoring system because it is impossible to work with an external database.

To solve this issue, two main approaches were studied, which are (1) TCP / IP socket communication and (2) the Web page parsing approach.

The first approach, which uses TCP / IP socket communication, has two drawbacks. The TCP / IP socket communication method does not directly access the built database. In addition, as the input and output is limited to a one-to-one communication, multiple users cannot obtain data at the same time and monitoring is limited.

The second approach, a Web page using PHP, returns a resulting value for a specified query. This approach has the advantage that multiple people can access. The Web page immediately, unlike TCP / IP communications.

The ultimate goal of our system is to grant ubiquitous access to the Web page universally. The design of our system must allow for accessing particular device tags and their corresponding information such as their table name, date, and other information. To address these considerations, a GET method was used.

In Fig. 3, information on tank COT01 and its corresponding Web address are shown. The data was accessed on 20 November 2012 and its contents were embedded.

http: localhost:8080 water_tank_query php

?table $=$ tank stame $=$ COT 01 s date $=\mathbf{2 0 1 2 - 1 1 - 2 0}$

Tank_name, Date, Temp_L, Temp_M, Temp_H, Level, Pressure

COT01, 2012-11-20 10:49:01, 22, 24, 28,5,12

COT01, 2012-11-2010+49:03,21, 24, 28,5,12

COT01, 2012-11-2010:49:05,21, 28, 31, - .12

Fig. 3. Water tank database data extraction

\section{B. Application Implementation}

The real-time monitoring system, “Tank boy, ” proposed in this paper aims to implement the smart phones application. The user run application first, application will show dialog message to receive login information. Application brings information to a device in the server by using the information from the user. Second, Application shows the device list, this list obtained by applying the normal range of each device. The list has been implemented for different ways to visualize device by using the Select button.

In addition to accessing the database from a system, considerations for the visualization implementation exist. This is known as application user interface synchronization.

Fig. 4 shows the tank data visualization using our approach. If the internal value of the activity is updated at certain time intervals, Android uses threads by default. Fig. 4 shows the current tank level, temperature, and pressure simultaneously using separate user interface visualization. Properly applied to quantify that value accordingly if you use the default thread, but the water level data, despite changes fixed the 
problem occurs.

Method was used to provide the android UI thread to solve this problem. Our system used asyncTask to update the UI, and a change in the parameters was reflected in the values in the database.

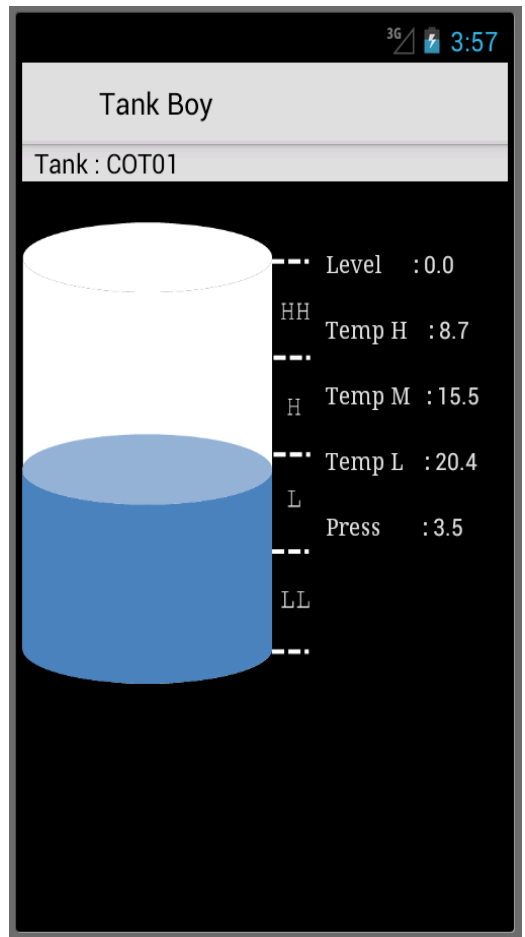

Fig. 4. Monitoring system example (Tank data visualization)

Fig. 5 shows the valve data visualization. Additionally, the system also shows the failure state.

Fig. 6 shows a visualization of the pump data; however, this system is yet to be implemented. Progress has been made with regards to this user interface and we are currently in the process of determining whether it is possible to implement the interface without using a separate thread.

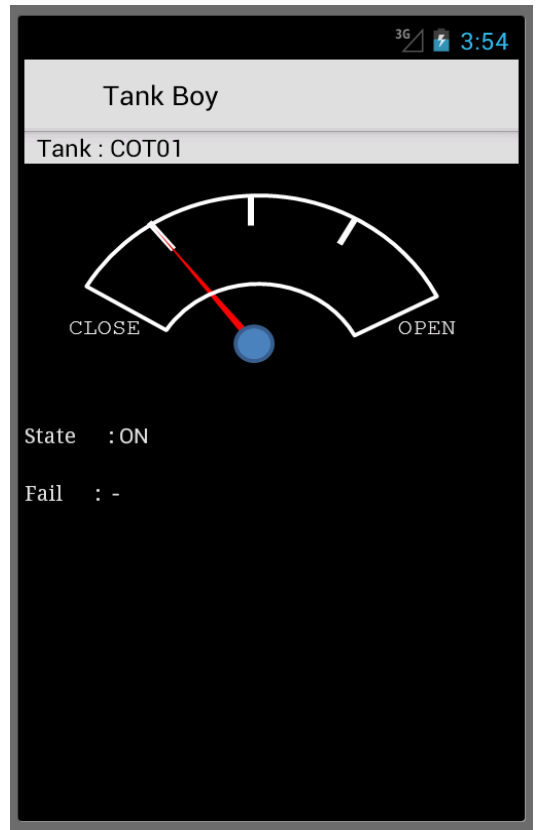

Fig. 5. Monitoring system example (Valve data visualization)

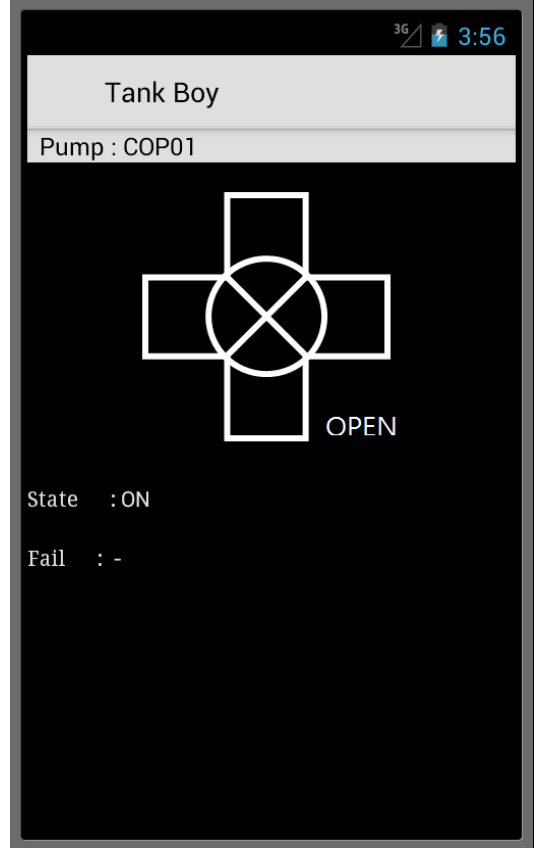

Fig. 6. Monitoring system example (Pump data visualization)

\section{CONCLUSION AND FURTHER WORKS}

This paper proposed a water tank monitoring and visualization system using smart-phones known as "Tank boy." This system is a real-time monitoring system using a database to establish a marine communication system. "Tank boy" works anywhere and anytime. Earlier, this system was restricted to a $\mathrm{PC}$ environment. However, its implementation on a smart-phone environment is novel.

The sensor data in our current database incurs a slight delay; this delay is due to two factors. The first is the delay in accessing the Web page. The second is due to the monitoring of the parsing information. Due to these restrictions, data cannot be transmitted in real time. These issues can be overcome with an improvement in the infrastructure of the telecommunication systems and an enhancement in the computing power of smart-phones.

This system proposed in this paper provides only a simple monitoring system. In future works, we expect to develop a system that can provide an analysis of the events that occur and use this to predict the lifetime of the sensors inside the ship.

We want to use data mining techniques in order to proceed with the research. Through this data, statistical analysis and modeling can be performed and useful patterns and relationships can be extracted [6].

Using data mining analysis module, the device data in database is changed to the new data. Also data mining analysis module used for monitoring data was stored without leverage, the dependent variable used in the life prediction is generated.

Our system will be completed from matching process failure and accident cases. And then, system is improving to warn about the risk of accidents and visualize detail information about tanks, valve, and pumps of remaining life information [7]. 


\section{REFERENCES}

[1] V. Chan, P. Ray, and N. Parameswaran S. Chen, "Mobile e-Health monitoring: an agent-based approach," IET Commun, vol. 2, pp. 223-230, 2008.

[2] S. Dai, and Y. Zhang, "A Wireless Physiological Multi-parameter Monitoring System Based on Mobile Communication Networks," in Proc. Computer-Based Medical Systems, 2006. CBMS 2006. 19th IEEE International Symposium on, Utah, 2006, pp. 473-478.

[3] S. Harries, F. Tillig, M. Wilken, and G. Zaraphonitis, "An integrated approach for simulation in the early ship design of a tanker," in Proc 10th International Conference on Computer and IT Applications in the Maritime Industries, Berlin, May 2011, pp. 411-425.

[4] D. Schwabe and G. Rossi, "An object oriented approach to web-based applications design," Theory and Practice of Object Systems, vol. 4, issue. 4, pp. 207-225.

[5] Two thirds of new mobile buyers now opting for smartphones. (July 1 2, 2012). [Online]. Available:

http://blog.nielsen.com/nielsenwire/online_mobile/two-thirds-of-newmobile-buyers-now-opting-for-smartphones/

[6] V. Ganti, J. Gehrke, and R. Ramakrishnan, "DEMON: mining and monitoring evolving data," IEEE Transactions on Knowledge and Data Engineering, vol. 13, pp.50-63, 2001.

[7] D. A. Keim, "Information visualization and visual data mining," IEEE Transactions on Visualization and Computer Graphics, vol. 8, pp. 1-8, 2002.

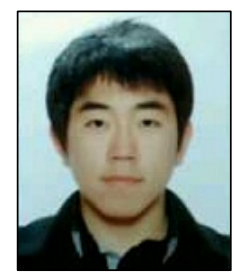

Haesung Tak is an undergraduate student in Pusan National University. His research interests are data parsing and reply analysis.

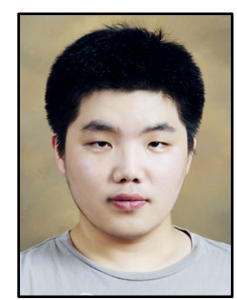

Daegun Kwon is an undergraduate student in Pusan National University. His research interests are basic algorithm, data mining, and graph drawing.

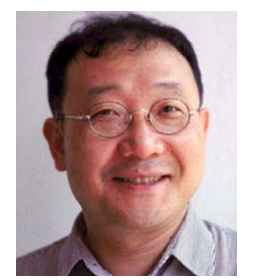

Hwan-Gue Cho is a Professor in Pusan National University. He received the B.S. degree from Seoul National University, Korea, and the M.S and Ph.D. degrees from Korea Advanced Institute of Science and Technology, Korea. His research interests are computer algorithms and bioinformatics. 